\title{
Non-destructive 3D Topography Modeling Based on Surface Profile Measurement Data
}

\author{
Mingxia Kang*, Wencheng Tang \\ School of Mechanical Engineering, Southeast University, Nanjing, Jiangsu Province 210000, China. \\ * Corresponding author. Tel.: +86 3337803355; email: 101000185@seu.edu.cn \\ Manuscript submitted April 09, 2018; accepted July 6, 2018. \\ doi: 10.17706/ijcee.2018.10.4.291-301
}

\begin{abstract}
Due to the limitations on the size and properties of samples, most of the surface topography tests are destructive and the contrast test of the surface morphology before and after friction and wear of the same workpiece cannot be carried out by the topography instruments. In addition, the destructive test increases the test period and the research cost, as well. In order to solve the above problems, a non-destructive surface topography measurement method is proposed in this paper. The 3D surface topography model of the workpiece surface can be established by the processing of the surface height distribution characteristics obtained by the surface profiler. It includes the discrete point reorganization, concentric processing, moving average filtering, equal length processing and other pre-processing and the post-processing like regression analysis, polynomial interpolation. Compared with the surface topography obtained by the topography instrument, it is found that the 3D surface topography model established not only characterize the surface features such as dents, bulges, and scratches accurately and effectively but also avoid the complex sample preparation processes. What is more, the surface topography of the workpiece can be acquired without destructing and the comparison test of the surface topography before and after friction and wear of the same workpiece can be conducted.
\end{abstract}

Key words: Concentric processing, discrete point reorganization, equal-length processing, moving average filtering, polynomial interpolation.

\section{Introduction}

The surface topography is a general term for the surface geometric shape on the concept of the microscopic angle, and it is the characterization of surface height distribution features, including surface roughness, waviness, surface texture and shape error, etc. [1], [2]. The topography symbolizes the fingerprint of the workpiece surface and it can reveal the essential features of the surface more directly compared with the surface roughness or surface contour [3]. The grinding process can be optimized more effectively with research of the effect of grinding process on the surface quality for precision components, such as ball screws pair, linear guide pair and rolling bearings, etc. from the perspective of the surface topography [4], [5]. Moreover, the microstructures such as pits, bulges and scratches in surface topography are the key factors influencing the thickness of the lubricant film and the friction and wear features for the friction pair surface [6]-[8]. No matter the optimization of the grinding process or the analysis on friction and wear characteristics of the surface, the investigation of the surface topography is an inevitable trend. 
Therefore, how to obtain the surface topography of the workpiece is a very significant issue.

It has many ways to obtain the surface topography of the workpiece in the domestic and foreign researches. For example, Magdalena et al. [9] measured the surface topography of the wear surface and the machined surface by the CMM, white interference microscope (WLI), scanning electron microscope (SEM). Nyman P et al. [10] got the topography using the vertical scan interferometer in wet clutch applications and studied the effects of surface topography parameters on the friction characteristics of the clutch. Chen $\mathrm{C}$ et al. [11] measured a 3D microscopic topography of the grinding wheel surface through a high-power optical lens and eliminated the noise during the measurement of the grinding wheel surface through a Gaussian filter. Wang Y et al. [12] discussed the effect of preloading on the micro-morphology of the workpiece surface and the results of simulation analysis were verified through the microscopic topography of the surface. Costa HL et al. [13] investigated the dynamic effect of the SEM image of the steel ball surface on the microscopic wear process of wear particles. Compared to traditional two-dimensional techniques for measuring the surface topography, the atomic force microscopy (AFM) can be used to measure the 3D topography of worn abrasive particles. Wu J et al. [14] achieved the geometry and surface morphology of wear particles at the nanometer level using the atomic force microscopy (AFM).

Although there are many methods to gain the surface topography, whether the white light interferometer using optical principles or the scanning electron microscope with the principle of electron imaging and the AFM with probe contact principles, they all have the strict requirements on the shape, size, or attributes of the samples. The samples need destructing when measuring the surface topography and the preparation is complex and cumbersome. For example, Ma Y H et al. [15] observed the shape of a sample with a small size by the scanning electron microscope. However, the effect was not obvious when the sample has a large size. Different sample preparation methods were systematically evaluated by Österreicher J A et al. Such as mechanical polishing, etching of various reagents, and electrolytic polishing with different electrolytes [16]. A non-conductive sample is coated to passivate the surface when obtained the surface topography using an electron microscope [17]. Generally, the sample is cut to ensure that the thickness is within the allowable measurement range of the instruments. However, for precision parts such as ball screws pair, linear guide pair and rolling bearings, the destructive measurement methods to get the surface topography causes high costs and it is impractical to obtain the massive data of topography features. On the other hand, the samples with destructive measurement cannot be used for comparative tests when the effect of different surface topography on the friction and wear characteristics is studied thus the experimental data lack reference significance.

In order to solve the problems caused by destructive testing, Darafon A et al. [18] designed a grinding wheel positioning system (rotary mechanism) to form a non-contact and non-destructive three-dimensional grinding wheel scanning system. It is capable of obtaining the surface topography without destroying the grinding wheel. However, most of the workpieces have complex structures and the measured surface needs to be perpendicular to the lens. The design of the mechanical structure cannot solve the general problems. Therefore, a non-destructive test method is urgent for obtaining the surface topography.

\section{Methodology}

Through reconstructing the discrete points characterizing the height distribution of the surface extracted by the surface profiler, this paper proposes a method to obtain a high-precision surface topography without destructing the workpiece. The measurement of the discrete points is completed through the slip between the stylus and measured surface and its greatest advantage is that some complex features of the surface can be measured directly, such as holes, grooves. As the fingerprint of the workpiece surface, the topography is 
composed of the height distribution features of each point. The 3D surface topography model is established by reorganizing the discrete points with the height distribution features without destructing the workpiece and the principle is shown in Fig. 1.

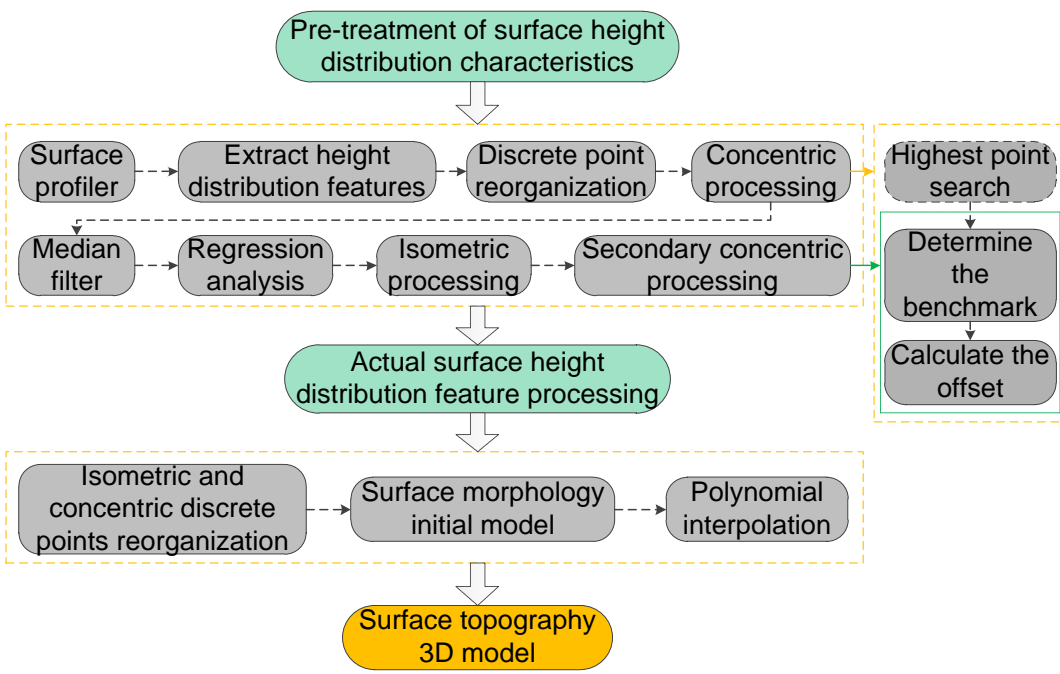

Fig. 1. Schematic diagram of 3D surface topography modeling.

\subsection{Pre-processing of Surface Topography Modeling}

\subsubsection{Extraction of the height distribution features}

The inner raceway surface of GZB45BA slider in roller heavy-duty linear guide pair is studied in this paper and the inner raceway surface topography model is built by the non-destructive method. In order to compare the surface topography of the raceway before and after wear, the measurement position is marked using a laser pen. The workpiece is placed on the stage and fixed by the magnetic stand. Setting the measuring range of the probe and the initial value of the lateral position. The height distribution features of the inner raceway surface are extracted using the surface profiler (Taylor Hobson), as shown in Fig. 2. The horizontal resolution of the probe is $0.125 \mathrm{um}$ and the resolution of the fine-tuning system for the lateral measurement is $20 \mathrm{um}$.

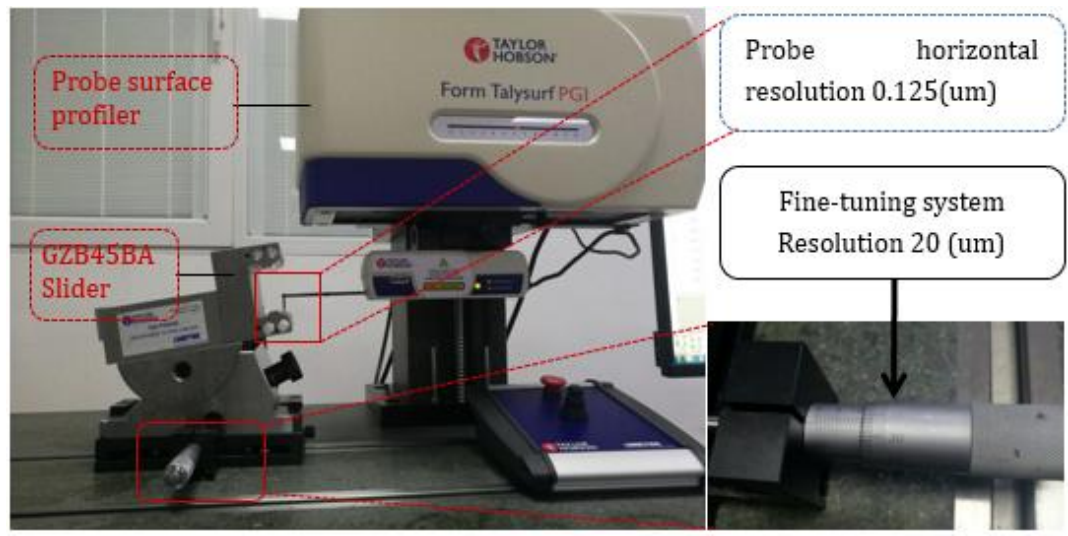

Fig. 2. Surface profiler.

In Fig. 3, it shows the extraction process of the surface height distribution features of the raceway. The measurement range is set to $15 \mathrm{~mm}$ to include the internal and external raceway surfaces of the slider reference plane. In experiments, the operations are performed within a range of 0-180 um in the lateral 
position with the measurement resolution $20 \mathrm{um}$, that is the entire extraction process is performed 10 times and all the measurement data are extracted and stored as a data basis for topography modeling.
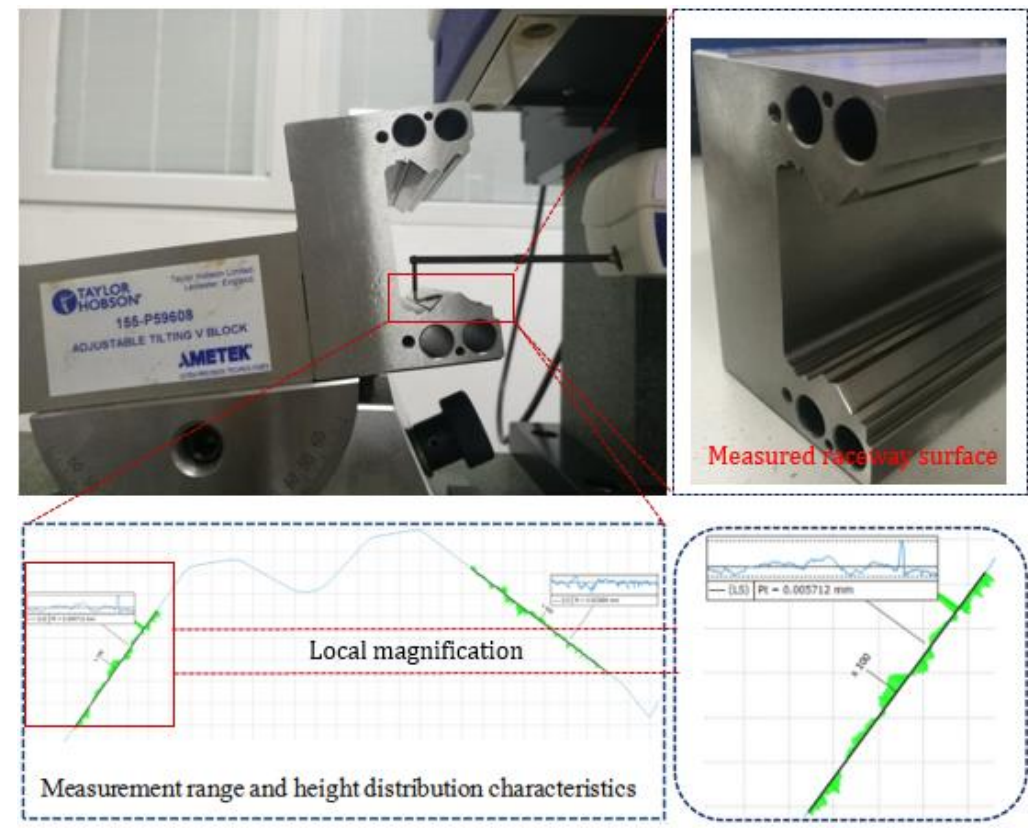

Fig. 3. Raceway surface height distribution feature extraction process.

\subsubsection{Discrete points recombination and concentric processing}

Two-dimensional recombination of the measurement data with the height distribution characteristics of the raceway surface is performed and the reorganization results are shown in Fig. 4. Since it is impossible to guarantee that the starting point is in the same position and the data length is equal when the probe measuring, the 10 recombination profiles are in different positions. Thus the concentric processing on discrete points is needed, that is, to coincident the contours. The concentric process consists of the search for the highest point of each set of data, the determination of the reference point, and the offset of the highest point. Taking the highest point of the first group as a benchmark and the remaining 9 groups of experimental data are coincided according to the offset of the vertical and horizontal coordinates between the highest point and the benchmark, as shown in Table 1. The highest point of each set of data after concentric processing is $(4355.125,2439.9869)$. This is why the measurement range is set to $15 \mathrm{~mm}$. It is to ensure that the highest position of the slider is always exist in each measurement and the concentric processing result is shown in Fig. 5.

Table 1. Concentric Process

\begin{tabular}{ccccc}
\hline \hline No. & The highest point coordinates & $\Delta y_{\text {offset }}$ & $\Delta x$ offset & $\begin{array}{c}\text { The coordinates after } \\
\text { concentric ontimization }\end{array}$ \\
\hline 1 & $(4355.125,2439.9869)$ & 0 & 0 & $(4355.125,2439.9869)$ \\
2 & $(4422.25,3472.8473)$ & 1032.860 & 67.125 & $(4355.125,2439.9869)$ \\
3 & $(4476.5,2727.166)$ & 287.1791 & 121.375 & $(4355.125,2439.9869)$ \\
4 & $(4397.375,2130.8599)$ & -309.127 & 42.25 & $(4355.125,2439.9869)$ \\
5 & $(4450.75,2781.6048)$ & 341.6179 & 95.625 & $(4355.125,2439.9869)$ \\
6 & $(4538.75,2770.9877)$ & 331.0008 & 183.625 & $(4355.125,2439.9869)$ \\
\hline
\end{tabular}




\begin{tabular}{lcccc}
\hline 7 & $(4643.5,3399.4883)$ & 959.5014 & 288.375 & $(4355.125,2439.9869)$ \\
8 & $(4522.125,2443.7699)$ & 3.783 & 167 & $(4355.125,2439.9869)$ \\
9 & $(4575.75,2422.6943)$ & -17.2926 & 220.625 & $(4355.125,2439.9869)$ \\
10 & $(4506.125,3040.8079)$ & 600.821 & 151 & $(4355.125,2439.9869)$ \\
\hline \hline
\end{tabular}

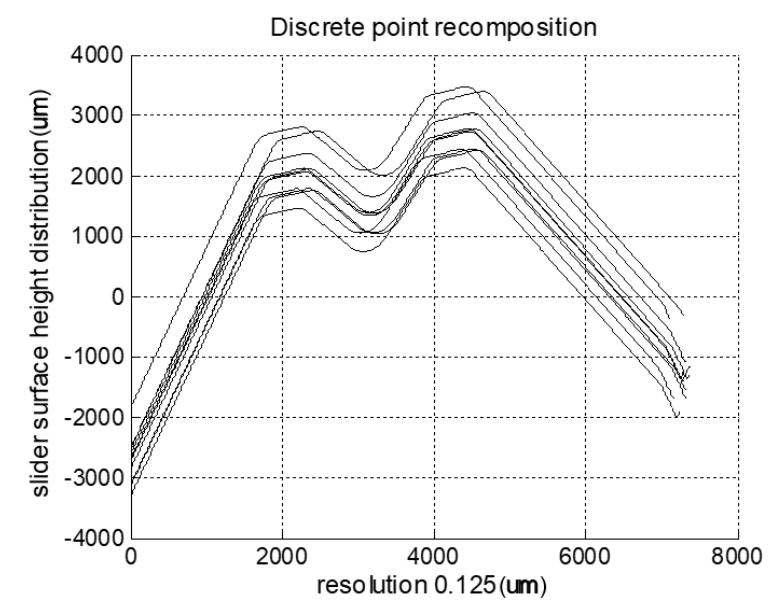

Fig. 4. 2D reorganization of discrete points.

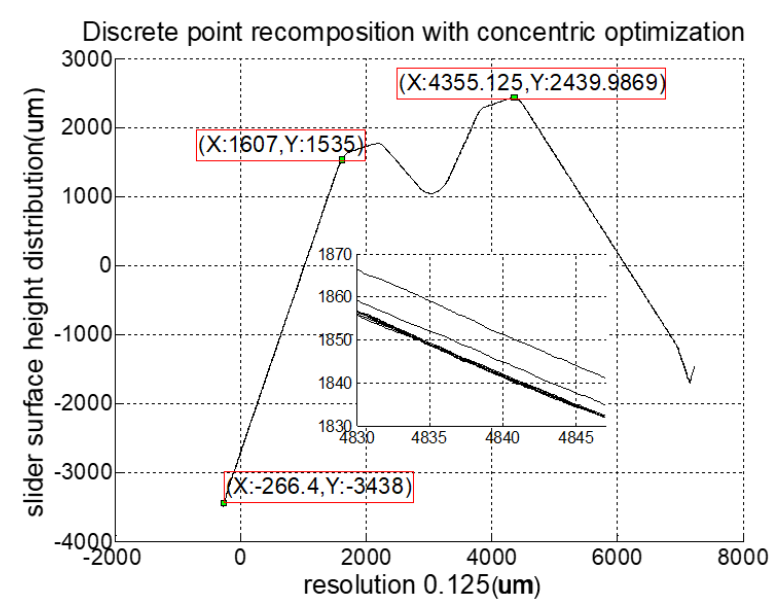

Fig. 5. 2D reorganization of discrete points after concentric processing.

\subsubsection{Median filtering, equal length processing and secondary concentric processing}

To avoid the effect of the external interference on the topography modeling, the median filtering [19] or moving average filtering [20] can be used before numerical analysis to remove noise. The smooth function is used to filter the 20 data specified around the data in this paper. And the variance of the filtered data is reduced from 0.6722 to 0.6223 with the little noise generated in data since the data is acquired by the probe and the profiler is placed on the anti-seismic platform.

To model the surface topography in a certain area of the workpiece, the equal length processing is performed on each set of data. Taking the first set of experimental data as a benchmark, the equal length data of each group is selected according to the difference of the abscissa serial number to the benchmark shown in Table 2. For example, the first group selects the data whose abscissa is 0 to 1500 , that is, the data with the abscissa number 1 to 12001 . The abscissa of the highest point in the second group is 35379 , then the abscissa number is $537+(1 \sim 12001)$ of the second isometric data by the offset. 
Table 2. Isometric Processing

\begin{tabular}{lllll}
\hline \hline No. & The highest point coordinates & $\begin{array}{l}\text { Highest point } \\
\text { abscissa number }\end{array}$ & $\begin{array}{c}\Delta X \\
\text { offset }\end{array}$ & $\begin{array}{l}\text { Isometric data } \\
\text { selection }\end{array}$ \\
\hline 1 & $(4355.125,2439.9869)$ & 34842 & 0 & $(1,12001)$ \\
2 & $(4422.25,3472.8473)$ & 35379 & 537 & $(538,12538)$ \\
3 & $(4476.5,2727.166)$ & 35813 & 971 & $(972,12972)$ \\
4 & $(4397.375,2130.8599)$ & 35180 & 338 & $(339,12339)$ \\
5 & $(4450.75,2781.6048)$ & 35607 & 765 & $(766,12766)$ \\
6 & $(4538.75,2770.9877)$ & 36311 & 1469 & $(1470,13470)$ \\
7 & $(4643.5,3399.4883)$ & 37149 & 2307 & $(2308,14308)$ \\
8 & $(4522.125,2443.7699)$ & 36178 & 1336 & $(1337,13337)$ \\
9 & $(4575.75,2422.6943)$ & 36607 & 1765 & $(1766,13766)$ \\
10 & $(4506.125,3040.8079)$ & 36050 & 1208 & $(1209,13209)$ \\
\hline \hline
\end{tabular}

Taking the first set of discrete points as a benchmark, the equal length data of each set of test is selected on the basis of concentric processing. Fig. 6 shows the reorganizations of the discrete points within the defined area. As the starting point of the measurement data in each test is not fixed, so the secondary concentric processing is required and the principle is the same with the concentric processing. It shows the coincide outline by the equal length processing and the secondary concentric processing in Fig. 7 .

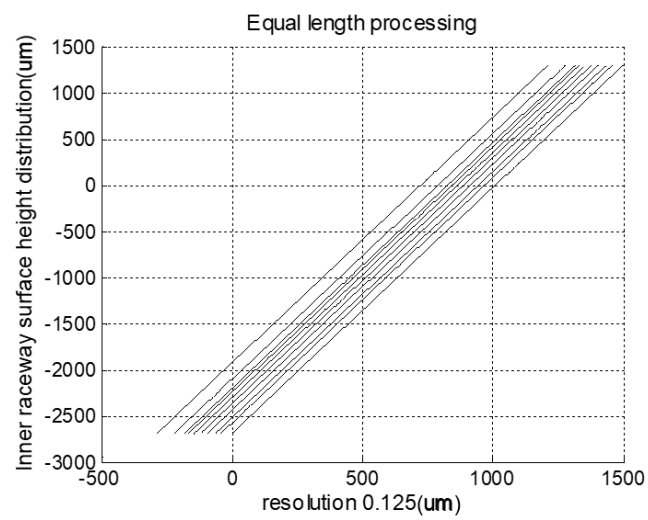

Fig. 6. Reorganization of discrete points processed by equal length.

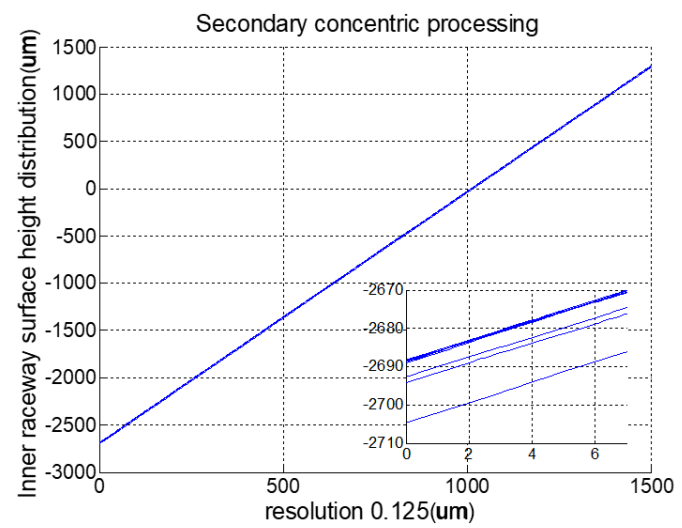

Fig. 7. Reorganization of discrete points processed by the secondary concentric processing. 


\subsection{The Establishment of the Surface Topography Model}

Regression analysis and polynomial interpolation are used to complete the modeling of the surface topography on basis of the pre-processing. In Fig. 5, it can be noticed that the measurement range of the inner raceway is $-266.4 \sim 1607$, thus the number of the collection point is 14987 according to the horizontal resolution $0.125 \mathrm{um}$. And 12001 discrete points of each experiment are selected for the subsequent surface topography modeling according to the equal length processing in Fig. 7.

\subsubsection{Regression analysis}

Fig. 7 shows that the surface of the inner raceway is flat. Thus, a linear regression analysis [21] is used to simulate the ideal surface of the inner raceway before friction and wear. The linear regression equation is determined by the first set of measurement data and the discrete points with height distribution characteristics of the ideal surface are determined by the regression equation. In Fig. 8, connect them to the actual discrete points measured by each set of experiments and it is the initial model of the inner surface of the raceway based on the real measurement data.

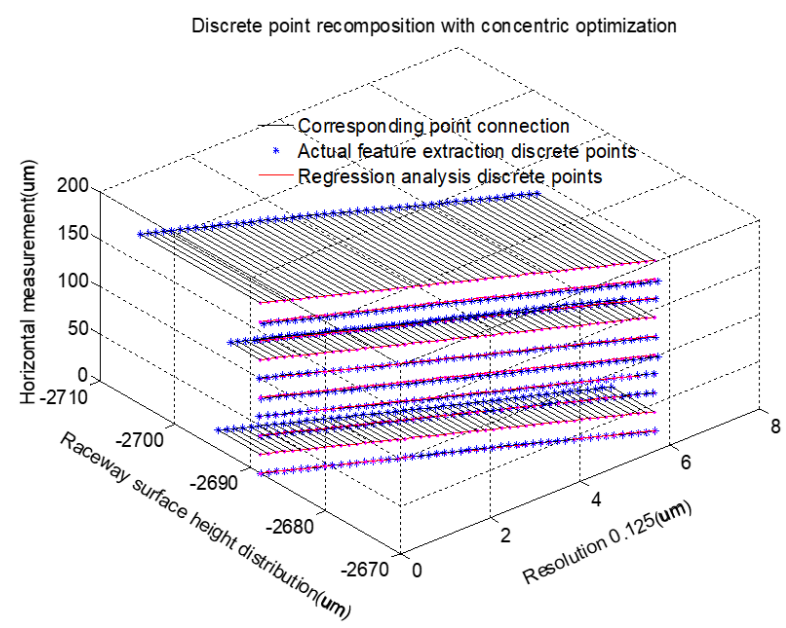

Fig. 8. Internal raceway surface topography initial model.

\subsubsection{Polynomial interpolation processing}

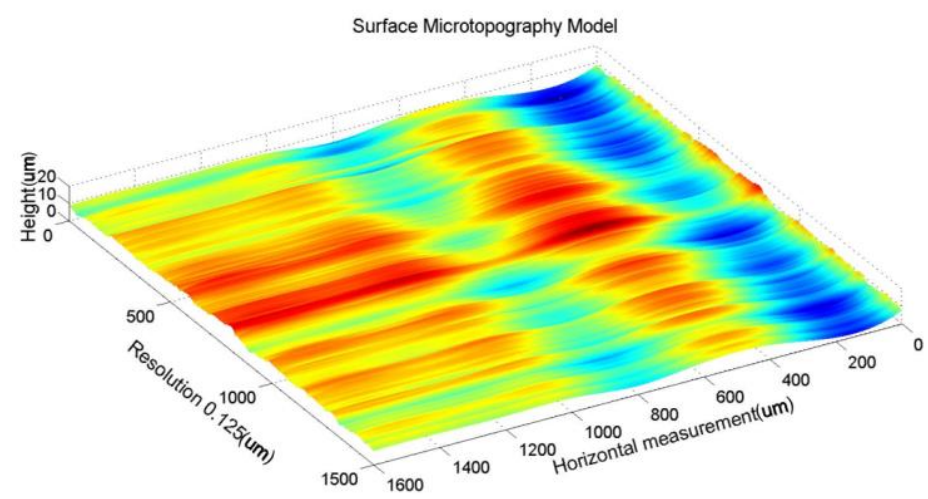

Fig. 9. 3D Surface topography model.

The real height value of the point is calculated from the measurement data and the attributes of the surface profiler. The value of the measurement data is $1.3 / 1000$ to the actual value for the discrete point. In order to facilitate the comparative analysis of subsequent experiments, the non-negative processing is 
performed, that is, all the data points are uniformly translated upward. And the three-dimensional model of the surface micro-topography is generated through the polynomial interpolation processing on the X-and Y-direction [22], as shown in Fig. 9.

\section{Experiment}

The surface topography is measured using the integrated topography module on the multi-functional friction and wear tester in this paper. And its working principle is the same as the white light interferometer. The surface of the inner raceway to be tested is cut to the allowable size of the sample by wire cutting and the flatness of the sample be ensured. Then the sample is rinsed with alcohol to remove the surface oil, debris and other substances. Besides that, it is wiped clean with industrial dust-free wiping paper and placed on the workbench. The measured surface is perpendicular to the lens, as shown in Fig. 10. The loading requirements of the workbench and the resolution of the objective lens require that the height of the measured sample should not exceed $7 \mathrm{~mm}$. Fig. 11 displays the surface topography of the inner raceway measured with the $10 x$ lens.
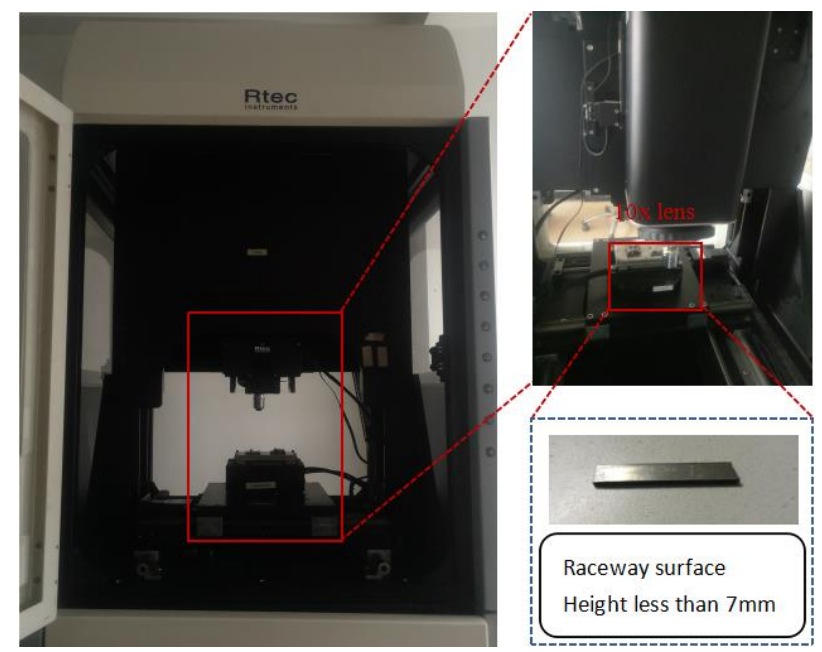

Fig. 10. Surface topography instrument.
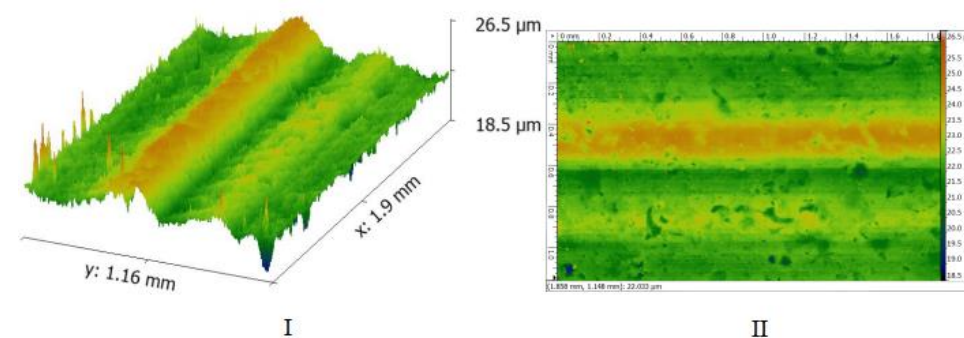

II

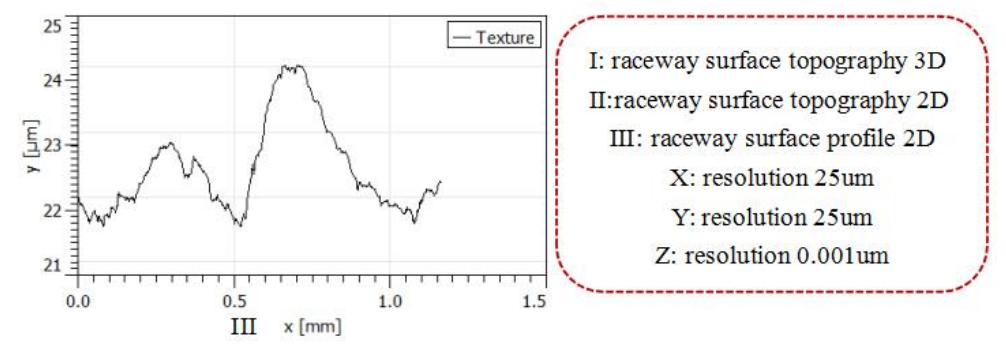

Fig. 11. 3D surface topography measured by the surface topography instrument.

The surface topography acquired by the topography instrument in Fig. 11 is compared with the three-dimensional topography model created by the non-destructive method in Fig. 9 and it is found that 
the three-dimensional topography model established can also characterize topography features like grooves, bulges, scratches, etc. [23] completely and accurately, and the resolution in X, Y-direction is higher than the topography instrument.

\section{Conclusion}

As the destructive detection methods are not suitable for some workpieces with high production cost and complicated process. It hinders the progress in the research of process optimization or frictional wear characteristics with surface morphology as the main factor. It proposes a three-dimensional topography modeling method based on measurement data by the surface profiler without destructing the workpiece. The principles of the method include the pre-processing of data like discrete point reorganization, concentric processing, moving average filtering, equal-length processing, secondary concentric processing and the post-processing like regression analysis and polynomial interpolation, as depicted in Fig. 1.

The height distribution features of the inner raceway of the slider are extracted by the surface profiler, and the three-dimensional topography model of the raceway surface is built by the non-destructive method. At the same time, the slider is wire-cut to obtain the raceway plane and the surface topography is acquired using the integrated topography module on the friction and wear tester. Comparing the three-dimensional surface topography established by the non-destructive modeling method and the topography instrument, respectively, it is found that the three-dimensional surface topography obtained by the non-destructive modeling method characterize the topography features of the workpiece surface like dents, bumps, and scratches accurately and effectively as well. It does not require complicated sample preparation processes and it is also suitable for non-conductive samples. At the same time, the advantage of this non-destructive method can be used to compare the topography of the same workpiece before and after wear, and it is of great significance to the research on the friction and wear characteristics of the workpiece surface based on the surface topography.

\section{Acknowledgment}

This work was financially Science Foundation of China (51675100) and National CNC Equipment Major Project (2016ZX04004008).

\section{References}

[1] Inami, T., Tanimoto, Y., Yamaguchi, M., et al. (2016). Surface topography, hardness, and frictional properties of GFRP for esthetic orthodontic wires. J Biomed Mater Res B Appl Biomater, 104(1), 88-95.

[2] Lipiński, D., \& Kacalak, W. (2016). Metrological aspects of abrasive tool active surface topography evaluation. Metrology \& Measurement Systems, 23(4), 352-361.

[3] Hu, S., Brunetiere, N., Huang, W., et al. (2017). Bi-gaussian surface identification and reconstruction with revised auto-correlation functions. Tribology International, 110, 185-194.

[4] Li, H. N., Yu, T. B., Zhu, L. D., et al. (2017). Analytical modeling of ground surface topography in monocrystalline silicon grinding considering the ductile-regime effect. Archives of Civil \& Mechanical Engineering, 17(4), 880-893.

[5] Setti, D., Kirsch, B., \& Aurich, J. C. (2017). A method to predict and visualize the wheel and work surface topography in surface grinding. Applied Mechanics and Materials, (7), 9-17.

[6] Zapletal, T., Sperka, P., Krupka, I., et al. (2017). The effect of surface grooves on transition to mixed lubrication. Tribology International, 114, 193-201.

[7] Lorentz, B., \& Albers, A. (2013). A numerical model for mixed lubrication taking into account surface topography, tangential adhesion effects and plastic deformations. Tribology International, 59, 259-266. 
[8] Krupka, I., Sperka, P., \& Hartl, M. (2016). Effect of surface roughness on lubricant film breakdown and transition from EHL to mixed lubrication. Tribology International, 100, 116-125.

[9] Niemczewska-Wójcik, M., \& Piekoszewski, W. (2017). The surface topography of a metallic femoral head and its influence on the wear mechanism of a polymeric acetabulum. Archives of Civil \& Mechanical Engineering, 17(2), 307-317.

[10] Nyman, P., Mäki, R., Olsson, R., et al. (2006). Influence of surface topography on friction characteristics in wet clutch applications. Wear, 261(1), 46-52.

[11] Chen, C., Tang, J., Chen, H., et al. (2017). Research about modeling of grinding workpiece surface topography based on real topography of grinding wheel. International Journal of Advanced Manufacturing Technology, (4), 1-11.

[12] Wang, Y., Xiu, S., \& Sun, C. (2017). Study on surface topography of workpiece in prestress dry grinding. International Journal of Advanced Manufacturing Technology, (1), 1-11.

[13] Costa, H. L., Ardila, M. A. N., Labiapari, W. S., et al. (2015). Effect of surface topography on the dynamics of the abrasive particles during micro-abrasion. Wear, 324-325, 129-139.

[14] Wu, J., \& Peng, Z. (2013). Investigation of the geometries and surface topographies of UHMWPE wear particles. Tribology International, 66(66), 208-218.

[15] Ma, Y. H., Zhang, X. H., \& Chen, X. G. (2013). Observation on non-conductive powder samples by scanning electron microscope. Applied Mechanics \& Materials, 320, 226-229.

[16] Österreicher, J. A., Kumar, M., Schiffl, A., et al. (2016). Sample preparation methods for scanning electron microscopy of homogenized Al-Mg-Si billets: a comparative study. Materials Characterization, 122, 63-69.

[17] Moser, R. D., \& Weiss, C. A. (2015). Sample preparation of nano-sized inorganic materials for scanning electron microscopy or transmission electron microscopy. Scientific Operating Procedure SOP-P-2, 37(4), 72-79.

[18] Darafon, A., Warkentin, A., \& Bauer, R. (2013). Characterization of grinding wheel topography using a white chromatic sensor. International Journal of Machine Tools \& Manufacture, 70(7), 22-31.

[19] Roy, A., Singha, J., Manam, L., et al. (2017). Combination of adaptive vector median filter and weighted mean filter for removal of high-density impulse noise from colour images. IET Image Processing, 11(6), 352-361.

[20] Kweon, S. J., Shin, S. H., Jo, S. H., et al. (2017). Reconfigurable high-order moving-average filter using inverter-based variable transconductance amplifiers. IEEE Transactions on Circuits \& Systems II Express Briefs, 61(12), 942-946.

[21] Lee, T., Ouarda, T. B. M. J., \& Yoon, S. (2017). KNN-based local linear regression for the analysis and simulation of low flow extremes under climatic influence. Climate Dynamics, (7), 1-19.

[22] Benachour, E., Draoui, B., Imine, B., et al. (2017). Numerical simulation of conjugate convection combined with the thermal conduction using a polynomial interpolation method. Advances in Mechanical Engineering, 9(5), 16878-16887.

[23] Sperka, P., Krupka, I., \& Hartl, M. (2016). The effect of surface grooves on film breakdowns in point contacts. Tribology International, 102, 249-256.

Mingxia Kang received her B.S. and M.Sc. degrees in mechanical engineering from Hohai University in 2014 and 2016 respectively. At present, she is a Ph.D. student in mechanical engineering at the School of Mechanical Engineering of Southeast University. Her main research direction is modeling the three-dimensional topography of the workpiece surface under the grinding process. 
Wencheng Tang received the B.S. degree from Nanjing Institute of Technology in 1982, the M.Sc. and the Ph.D. degrees from Southeast University in 1987. From 2008 to 2014, he was the dean of the School of Mechanical Engineering. His main research directions are CAD/CAE/CAM, computer integrated manufacturing system, researches on friction and wear of functional components and static and dynamic analysis and optimization design of structural parts. 